\title{
Empiemas pleurales por Gemella spp: una etiología no tan infrecuente
}

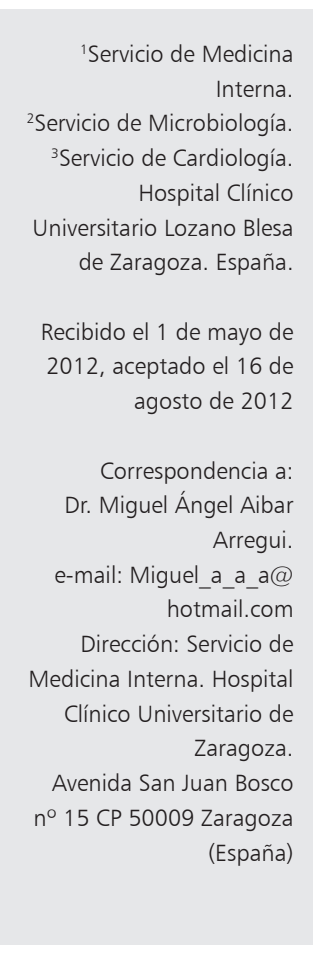

\author{
MIGUEL ÁNGEL AIBAR-ARREGUI' ${ }^{1}$, BEGOÑA DE ESCALANTE-YANGÜELA ${ }^{1}$, \\ ANA GARRIDO-BUENACHE², MARÍA ELENA NAVARRO-AGUILAR ${ }^{1}$, \\ JOSÉ MONTOYA-ARENAS ${ }^{3}$, MARÍA DEL MAR RODERO-ROLDÁN ${ }^{1}$
}

\section{Pleural empyema due to Gemella spp. Report of 12 cases}

\begin{abstract}
Background: Gemella genus bacteria can produce localized or generalized severe infections, but very rarely they have been described as causing pulmonary infections or pleural empyemas. Aim: To characterize patients with empyema caused by Gemella genus bacteria. Material and Methods: The database of a Microbiology laboratory of a Spanish hospital was reviewed, searching for Gemella positive cultures of pleural effusions in a period of five years. Results: We identified 12 patients (11 males) with Gemella spp pleural empyema. Eight were infected with G. haemolysans and four with G. morbillorum. All patients had predisposing factors such as poor oral hygiene, smoking, chronic cardiovascular or respiratory disease, alcoholism or malignancies. In ten cases, a thoracic drainage tube was placed with fibrinolysis in seven. One patient needed surgery because of a relapse of the empyema. Two patients died because of an advanced neoplasm, and the empyema was resolved in the rest. Conclusions: Gemella pleural empyema can occur and its isolation must not be seen as a contamination.

(Rev Med Chile 2012; 140: 1544-1547).
\end{abstract}

Key words: Empyema, pleural; Gemella; Lung diseases.
$\mathrm{E}$ n los últimos años estamos asistiendo a un aumento de las infecciones por gérmenes oportunistas y de infecciones previamente consideradas como poco frecuentes. Esto es debido en parte al envejecimiento de la población pero también al mayor número de actuaciones intervencionistas, dispositivos intravasculares, trasplantes y tratamientos inmunomoduladores, entre otros; sin olvidarnos del papel de la infección por el virus de inmunodeficiencia humana (VIH) ${ }^{1,2}$.

Entre estos gérmenes se encuentran las bacterias del género Gemella, cocos Gram positivos, anaerobios facultativos, que forman parte de la flora comensal habitual de la orofaringe, tracto digestivo y urinario y que en determinadas circunstancias pueden producir infecciones graves tanto a nivel local como generalizadas ${ }^{3,4}$.

Entre las infecciones que se han atribuido a $\mathrm{Ge}$ mella spp destacan las endocarditis, pero también se han descrito infecciones articulares, sepsis, abs- cesos cerebrales, pulmonares y empiemas pleurales así como infecciones graves del tracto urinario ${ }^{3}$.

En la mayor parte de los casos descritos se ha encontrado algún factor predisponente para la infección como pueden ser la mala higiene dental, cirugías digestiva u oral, diabetes, enfermedades neurológicas crónicas, insuficiencia renal, vasculitis, alcoholismo, uso de drogas por vía parenteral y enfermedades cardiovasculares o pulmonares crónicas $^{3-5}$.

En la revisión de la literatura, la mayor parte de las publicaciones corresponden a casos clínicos aislados o series reducidas de casos, particularmente en lo que se refiere a infecciones pleuropulmonares por Gemella, sin que existan datos sobre prevalencia o incidencia de dichas infecciones.

En el presente trabajo se describen los casos de empiema pleural por Gemella spp diagnosticados en un hospital de referencia de Zaragoza, España, a lo largo de cinco años. 
Empiema pleural por Gemella spp - M. A Aibar-Arregui et al

\section{Métodos}

Para la realización de este trabajo se han revisado las bases de datos del Servicio de Microbiología del Hospital Clínico Universitario "Lozano Blesa" para localizar todos los casos de empiema pleural por Gemella spp diagnosticados entre el 1 de septiembre de 2006 y el 1 de septiembre de 2011. De cada paciente se han extraído los datos de filiación, cuadro clínico, exámenes complementarios, diagnóstico microbiológico y bioquímico en líquido pleural así como el tratamiento instaurado y la evolución clínica.

Los cultivos se realizaron en agar sangre y la identificación de las especies de Gemella se realizó mediante el sistema API 20 STREP ${ }^{\circledR}$. El antibiograma se obtuvo mediante disco-placa en la mayoría de los casos (9 de 12 casos); en dos casos se realizó mediante la técnica de Wider ${ }^{\circledR}$ (Soria-Melguizo) y en un único caso no fue posible la realización del antibiograma.

\section{Resultados}

En los cinco años revisados se han encontrado un total de 12 casos de empiema por gérmenes del género Gemella; 8 por G. haemolysans y 4 por
G. morbillorum. Representan $0,81 \%$ del total de aislamientos de cultivos pleurales en nuestro centro en este período (1.482). De los 12 pacientes, once eran hombres y una mujer con edades comprendidas entre los 28 y 83 años. En cuanto a los servicios de referencia, el que más casos aportó fue el Servicio de Medicina Interna con cinco casos, seguido del Servicio de Neumología con tres casos y los Servicios de Digestivo, Infecciosas, Cirugía Torácica y Oncología con un caso cada uno. Sólo tres pacientes precisaron tratamiento en UCI en algún momento de su evolución (casos 3, 6 y 7) y uno de ellos reingresó tras el alta hospitalaria por recidiva de la infección (caso 3). La información clínica y los exámenes de laboratorio complementarios realizados pueden consultarse en la Tabla 1.

En la totalidad de los pacientes se encontró algún factor predisponente para la infección, siendo los más frecuentes el tabaquismo y el consumo de alcohol; en cuatro casos se realizó estudio de infección por el VIH siendo negativo en todos los casos.

El tratamiento antibiótico más utilizado fue la combinación de amoxicilina con ácido clavulánico a dosis de $1 \mathrm{~g}-200 \mathrm{mg}$ cada $8 \mathrm{~h}$ por vía intravenosa (Tabla 2); en todos los casos se procedió a la evacuación del líquido pleural, precisando siete pacientes la administración de agentes fibrinolíticos

Tabla 1. Datos clínicos y de pruebas complementarias

\begin{tabular}{|c|clllll|}
\hline Caso & $\begin{array}{c}\text { Edad / } \\
\text { Sexo }\end{array}$ & Antecedentes & Cuadro clínico & $\begin{array}{l}\text { Radiografía de } \\
\text { tórax }\end{array}$ & $\begin{array}{c}\text { HC } \\
\text { Otras } \\
\text { pruebas }\end{array}$ \\
\hline 1 & $46 \mathrm{H}$ & T, OH, HDA & Disnea, fiebre & DP derecho & NR & TAC \\
\hline 2 & $77 \mathrm{H}$ & T, EPOC, cáncer pulmón & Disnea, tos, expectoración & Neumonía + DP Dcho & NR & TAC \\
\hline 4 & $45 \mathrm{H}$ & T, OH, boca séptica & Disnea, tos, fiebre & Neumonía + DP Dcho & Neg & TAC \\
\hline 5 & $63 \mathrm{H}$ & Diabetes mellitus & Febrícula & Neumonía + DP Dcho & NR & TAC \\
\hline 6 & $64 \mathrm{M}$ & OH, T, B. séptica, IRC & Tos, fiebre & DP Izquierdo & NR & TAC, FBC \\
\hline 7 & $66 \mathrm{H}$ & Tabaquismo & Disnea, ascitis, edema & DP Derecho & NR & NR \\
\hline 9 & $28 \mathrm{H}$ & OH, Cocaína, Speed & Tos, disnea & Neumonía + DP Izdo & Neg & TAC, FBC \\
\hline 10 & $73 \mathrm{H}$ & EPOC, T, cáncer pulmón & Tos, fiebre, disnea & Neumonía + DP Izdo & Neg & NR \\
\hline 11 & $66 \mathrm{H}$ & Diabetes, EPOC & Tos, disnea & DP Derecho & Neg & TAC, FBC \\
\hline 12 & $72 \mathrm{H}$ & T, OH, UDVP, VHC & Tos, disnea, expectoración & DP Izquierdo & Neg & TAC \\
\hline
\end{tabular}

H: Hombre; M: Mujer; HC: Hemocultivos; T: Tabaquismo; OH: Alcoholismo; HDA: Hemorragia digestiva alta; DP: Derrame pleural; Neg: Negativos; B. Séptica: Boca séptica; IRC: Insuficiencia renal crónica; EPOC: Enfermedad pulmonar obstructiva crónica; UDVP: Usuario de drogas por vía parenteral; VHC: Virus Hepatitis C; QT: Quimioterapia; N: Neumonia; Dcho: Derecho; Izdo: Izquierdo; NR: No realizado; TAC: Tomografía axial computarizada de tórax; FBC: Fibrobroncoscopia. 
Tabla 2. Datos bioquímicos, microbiológicos y tratamientos

\begin{tabular}{|c|c|c|c|c|c|c|c|c|c|c|c|}
\hline \multirow[t]{2}{*}{ Caso } & \multicolumn{7}{|c|}{ Bioquímica de líquido pleural } & \multirow{2}{*}{$\begin{array}{c}\text { Cultivo } \\
\text { (n) }\end{array}$} & \multirow{2}{*}{$\begin{array}{l}\text { Antibió- } \\
\text { tico }\end{array}$} & \multirow[t]{2}{*}{ Drenaje } & \multirow[t]{2}{*}{ Evolución } \\
\hline & $\mathbf{M}$ & pH & $\begin{array}{l}\text { Proteí- } \\
\text { nas }\end{array}$ & $\begin{array}{l}\text { Glu- } \\
\text { cosa }\end{array}$ & $\begin{array}{l}\text { Leuco- } \\
\text { citos } \\
(\%)\end{array}$ & LDH & ADA & & & & \\
\hline 1 & $S$ & ND & ND & ND & ND & ND & ND & $\begin{array}{c}\text { G. haemolysans } \\
\text { (3) }\end{array}$ & $\begin{array}{l}\text { Vancomicina } \\
\text { Clindamicina }\end{array}$ & $\begin{array}{c}\text { TT + } \\
\text { Fibrinolisis }\end{array}$ & Curación \\
\hline 2 & $S$ & 7,24 & 3,9 & 99 & $\begin{array}{l}360 \\
(16)\end{array}$ & 5.044 & 17 & $\begin{array}{l}\text { G. morbillorum } \\
\text { (1) }\end{array}$ & $\begin{array}{c}\text { AmoxiClavu- } \\
\text { lánico }\end{array}$ & $\begin{array}{l}\text { Toracocen- } \\
\text { tesis }\end{array}$ & Exitus \\
\hline 3 & $P$ & 6,8 & 3,9 & 2 & $\begin{array}{c}68.800 \\
(80)\end{array}$ & 10.938 & 145 & $\begin{array}{c}\text { G. haemolysans } \\
\text { (4) }\end{array}$ & $\begin{array}{l}\text { Ciprofloxa- } \\
\text { cina }\end{array}$ & $\begin{array}{l}\text { TT + } \\
\text { Cirugía }\end{array}$ & Curación ${ }^{\dagger \ddagger}$ \\
\hline 4 & $S$ & 6,7 & ND & ND & ND & ND & ND & $\begin{array}{c}\text { G. haemolysans } \\
\text { (2) }\end{array}$ & $\begin{array}{c}\text { AmoxiClavu- } \\
\text { lánico }\end{array}$ & $\begin{array}{c}\text { TT + } \\
\text { Fibrinolisis }\end{array}$ & Curación \\
\hline 5 & $P$ & ND & ND & ND & ND & ND & ND & $\begin{array}{c}\text { G. haemolysans } \\
\text { (1) }\end{array}$ & $\begin{array}{l}\text { AmoxiClavu- } \\
\text { lánico }\end{array}$ & $\begin{array}{c}\text { TT + } \\
\text { Fibrinolisis }\end{array}$ & Curación \\
\hline 6 & S & 7,8 & ND & ND & ND & ND & ND & $\begin{array}{c}\text { G. morbillorum } \\
\text { (1) }\end{array}$ & Ceftriaxona & $\begin{array}{l}\text { Tubo de } \\
\text { tórax }\end{array}$ & Curación ${ }^{\dagger}$ \\
\hline 7 & $\mathrm{~S}$ & 7,6 & $<0,1$ & 17 & ND & 552 & ND & $\begin{array}{c}\text { G. morbillorum } \\
\text { (1) }\end{array}$ & $\begin{array}{l}\text { Piperacilina } \\
\text { Tazobactam }\end{array}$ & $\begin{array}{l}\text { Tubo de } \\
\text { tórax }\end{array}$ & Curación ${ }^{\dagger}$ \\
\hline 8 & $\mathrm{~S}$ & 7,5 & 5,3 & 4 & $\begin{array}{c}7.706 \\
(80)\end{array}$ & 5.059 & 98 & $\begin{array}{l}\text { G. haemolysans } \\
\text { (3) }\end{array}$ & $\begin{array}{c}\text { AmoxiClavu- } \\
\text { lánico }\end{array}$ & $\begin{array}{c}\text { TT + } \\
\text { Fibrinolisis }\end{array}$ & Curación \\
\hline 9 & $S$ & 6,8 & ND & 10 & $\begin{array}{c}240.000 \\
(93)\end{array}$ & 37 & 130 & $\begin{array}{l}\text { G. haemolysans } \\
\text { (1) }\end{array}$ & $\begin{array}{l}\text { Tobramicina } \\
\text { Ceftazidima }\end{array}$ & $\begin{array}{c}\text { TT + } \\
\text { Fibrinolisis }\end{array}$ & Curación \\
\hline 10 & $S$ & 7,9 & 2,2 & 96 & $\begin{array}{c}1.020 \\
(N D)\end{array}$ & 329 & ND & $\begin{array}{c}\text { G. haemolysans } \\
\text { (1) }\end{array}$ & Levofloxacina & $\begin{array}{c}\text { TT + } \\
\text { Fibrinolisis }\end{array}$ & Curación \\
\hline 11 & $S$ & 7,3 & 4,67 & 150 & $\begin{array}{c}1.351 \\
(25)\end{array}$ & 211 & 35 & $\begin{array}{c}\text { G. haemolysans } \\
\text { (1) }\end{array}$ & $\begin{array}{l}\text { AmoxiClavu- } \\
\text { lánico }\end{array}$ & $\begin{array}{c}\text { TT + Fibri- } \\
\text { nolisis }\end{array}$ & Curación \\
\hline 12 & $\mathrm{~S}$ & ND & ND & ND & ND & ND & ND & $\begin{array}{c}\text { G. morbillorum } \\
\text { (1) }\end{array}$ & $\begin{array}{c}\text { AmoxiClavu- } \\
\text { lánico }\end{array}$ & $\begin{array}{l}\text { Toracocen- } \\
\text { tesis }\end{array}$ & Exitus \\
\hline
\end{tabular}

M: Aspecto macroscópico; S: Seroso; P: Purulento; n: Número de muestras positivas; TT: Tubo de drenaje torácico; ND: No disponible; Unidades: Proteínas (g/dL), Glucosa (mg/dL), Leucocitos ( $\mathrm{mm}^{3}$ y \%), LDH (UI/L), ADA (UI/L). ${ }^{\dagger}$ Estancia en UCl; *Reingreso.

en el espacio pleural además de la colocación de tubo de drenaje. La evolución clínica fue satisfactoria en diez pacientes, falleciendo los otros dos casos, ambos en el contexto de neoplasias avanzadas.

\section{Discusión}

Los microorganismos del género Gemella son cocáceas Gram positivas, anaerobios facultativos y catalasa negativos; actualmente dentro de este género se encuentran G. haemolysans, G. morbillorum, G. bergeri, G. asacharolytica y G. sanguinis, aunque tan sólo los dos primeros se han identificado como patógenos potenciales para el ser humano ${ }^{3,4}$. La reciente reclasificación de ambos gérmenes, previamente conocidos como
Neisseria haemolysans y Streptococcus morbillorum, puede explicar en parte la escasez de estudios encontrados en la revisión de la literatura y, además, hay que tener en cuenta que las similitudes con otros géneros pueden llevarnos a infraestimar la prevalencia de infecciones por Gemella spp si no se realiza un estudio microbiológico completo ${ }^{4-6}$.

Hemos encontrado escasas referencias a infecciones pleuropulmonares por Gemella spp, y la mayoría de ellas pertenecen a casos aislados ${ }^{5,7-10} \mathrm{O}$ a series de casos entre las que destacan la de García y cols. ${ }^{3}$ y la de Senent y cols. ${ }^{4}$, por ser las únicas en las que aparece más de un caso de empiema pleural.

En nuestra serie, se han identificado factores predisponentes para la infección en la totalidad de los casos, siendo los más frecuentes el taba- 
quismo y el consumo excesivo de alcohol; no hemos encontrado ningún caso de infección por el VIH aunque sólo se había realizado despistaje en cuatro pacientes; tampoco hemos encontrado ningún paciente con tuberculosis activa por lo que nuestro trabajo no puede aportar datos a la posible relación entre ambas que se ha sugerido en otros estudios ${ }^{11,12}$.

En seis casos el empiema pleural era de origen paraneumónico mientras que en los otros seis no se ha identificado neumonía en las pruebas de imagen; de ellos un paciente tenía un cuerpo extraño intrabronquial que se extrajo por fibrobroncoscopia, cuatro tenían un hábito enólico muy importante por lo que la broncoaspiración puede considerase el mecanismo más probable para la infección, y el último presentaba una neoplasia esofágica sobre la que se había colocado recientemente una prótesis autoexpandible lo que, unido a la quimioterapia podría justificar la diseminación hematógena de la infección.

En cuanto al tratamiento instaurado en los pacientes de nuestra revisión, el antibiótico más usado de forma empírica fue amoxicilinaácido clavulánico. Aunque no es el tratamiento de elección (penicilina $\mathrm{G}$ asociada o no a un aminoglucósido) ni alternativo (vancomicina o un macrólido), en todos los casos resultó ser sensible en el antibiograma.

En los pacientes en los que se utilizaron otros antibióticos, la revisión de las historias clínicas sugiere que la decisión se tomó en base a comorbilidades o circunstancias particulares; por ejemplo, los dos pacientes tratados con quinolonas presentaban alergia a beta-lactámicos y en ambos se realizó un antibiograma que demostraba la sensibilidad del germen al tratamiento.

La evolución clínica fue satisfactoria en diez pacientes, produciéndose el fallecimiento de los otros dos en el contexto de su enfermedad neoplásica diseminada, por lo que, no se puede hablar propiamente de un fracaso terapéutico. Este buen comportamiento clínico coincide con los casos revisados en la literatura, sin embargo, un análisis más detenido nos demuestra que en siete casos se precisó la realización de fibrinolisis local para la resolución del cuadro y que el $25 \%$ de nuestros pacientes precisaron tratamiento en UCI en algún momento de la evolución, mientras que en otro caso se produjo una recaída que obligó a un nuevo ingreso hospitalario y cirugía posterior, lo que nos hace valorar con más cuidado la verdadera importancia de la infección.

En nuestra opinión, el empiema pleural por Gemella spp, si bien es poco habitual, no debe considerarse algo excepcional y no debe cometerse el error de asumir como una contaminación el aislamiento de este germen en muestras biológicas, sino realizar un estudio microbiológico completo y un tratamiento adecuado de la infección que garantiza, en la mayoría de los casos, la curación del enfermo.

\section{Referencias}

1. Jones KE, Patel NG, Levy MA, Storeygard A, Balk D, Gittleman JL, et al. Global trends in emerging infectious diseases. Nature 2008; 451 (7181): 990-3.

2. Desenclos JC, De Valk H. Med Mal Infect 2005; 35 (2): 49-61.

3. García-Lechuz JM, Cuevas-Lobato O, Hernangómez S, Hermida A, Guinea J, Marín M, et al. Extra-abdominal infections attributable to Gemella species. Int J Infect Dis 2002; 6: 78-82.

4. Senent C, Sancho JN, Chiner E, Signes-Costa J, Camarasa A, Andreu AL. Empiema pleural por microorganismos del género Gemella: una complicación infrecuente. Arch Bronconeumol 2008; 44: 574-7.

5. Canet JJ, Hernández R, Almagro P, Garau J. Empiema pleural por Gemella morbillorum. Enferm Infecc Microbiol Clin 2001; 19: 189.

6. Ruoff KL. Miscellaneous catalase-negative, gram-positive cocci: emerging opportunists. J Clin Microbiol 2002; 40: 1129-33.

7. Veziris N, Fuhrman C, Chouaid C, Marque E, Housset B, Lange J, et al. Empyema of the thorax due to Gemella haemolysans. J Infect 1999; 39: 245-6.

8. De Soyza A, Higgins B, Gould K. An inusual case of pulmonary abscess. J Infect 2000; 41: 114.

9. Signes Costa J, Chiner E, Arriero JM. Neumonía necrosante y empiema por Gemella morbillorum en un paciente laringuectomizado. Arch Bronconeumol 2000; 36: 601-2.

10. García del Busto A, Moreno R, Pardo F, Ferrándiz A. Empiema causado por Gemella morbillorum. Med Clin (Barc) 1995; 104: 196-7.

11. Maestre JR, Sánchez P, Buezas V, Roig F, Villegas F, Callol L. Cavidad tuberculosa abscesificada de evolución tórpida por sobreinfección con Gemella haemolysans. Enferm Infecc Microbiol Clin 2001; 19: 507-8.

12. Kondo M, Tanoue C, Yamamoto S, Shingu M. Enhanced growth of mycobacteria by culture filtrate of Gemella haemolysans. Kurume Med J 1990; 37: 141-7. 